\title{
Gestão do conhecimento: tipologia a partir dos fatores contextuais da organização
}

\author{
Knowledge management: Typology from \\ organizational contextual factors
}

\author{
Rodrigo Valio Dominguez GONZALEZ1 (iD) 0000-0003-0531-9638 \\ Manoel Fernando MARTINS² (D) 0000-0003-2787-5977 \\ Tatiana Massaroli MELO3 (D) 0000-0003-2787-7753
}

\section{Resumo}

A gestão do conhecimento como prática capaz de trazer vantagem competitiva e inovação às firmas é um consenso entre os pesquisadores. A gestão do conhecimento é alicerçada por fatores contextuais, desenvolvidos de forma distinta pelas organizações. Dessa forma, esta pesquisa tem o propósito de identificar uma tipologia quanto ao desenvolvimento desses fatores contextuais. $O$ estudo considera cinco constructos organizacionais relacionados à gestão do conhecimento: recursos humanos, trabalho em equipe, cultura organizacional, estrutura organizacional e desenvolvimento e absorção de conhecimento. A pesquisa é desenvolvida a partir de um survey em 78 empresas do setor automobilístico. A análise de dados é baseada nas técnicas estatísticas de análise fatorial exploratória, que desdobra as variáveis mensuráveis em fatores latentes, análise de cluster, que identifica agrupamentos de empresas, e análise discriminante, que valida a diferença estatística entre os clusters identificados. Os resultados apontam a existência de quatro agrupamentos distintos quanto à pratica da gestão do conhecimento, denominados de "Empresas Inovadoras", "Empresas Exploradoras", "Empresas Explotatoras" e "Empresas Retardatárias".

Palavras-chave: Análise de cluster. Análise fatorial. Fatores contextuais. Gestão do Conhecimento. Indústria automobilística.

\begin{abstract}
Knowledge Management as a practice that returns competitive advantage and innovation to firms is a consensus among researchers. The Knowledge Management is supported by contextual factors developed differently by organizations. Thus, the aim of this research was to identify a typology for the development of these contextual factors. The study considers five organizational constructs related to

1 Universidade Estadual de Campinas, Faculdade de Ciências Aplicadas. R. Pedro Zaccaria, 1300, 13484-350, Limeira, SP, Brasil. Correspondência para/ Correspondence to: R.V.D. GONZALEZ.E-mail:<rodrigo.gonzalez@fca.unicamp.br>.

2 Universidade Federal de São Carlos, Centro de Ciências Exatas e de Tecnologia, Departamento de Engenharia de Produção. São Carlos, SP, Brasil.

3 Universidade Estadual Paulista Júlio de Mesquita Filho, Faculdade de Ciências e Letras, Departamento de Ciências Econômicas. Araraquara, SP, Brasil.

Recebido em 27 de janeiro de 2017, versão final reapresentada em 11 de julho de 2017 e aprovado em 16 de agosto de 2017.

Apoio: Fundação de Amparo à Pesquisa do Estado de São Paulo (Processo n²016/024401-2) e Conselho Nacional de Desenvolvimento Científico e Tecnológico (Processo n 445205/2017-8).

Como citar este artigo/How to cite this article

Gonzalez, R. V. D.; Martins, M. F.; Melo, T. M. Gestão do conhecimento: tipologia a partir dos fatores contextuais da organização. Transinformação, v. 30 , n. 2, p. 249-266, 2018. http://dx.doi.org/10.1590/2318-08892018000200009
\end{abstract}


Knowledge Management: human resources, teamwork, organizational culture, organizational structure, and knowledge development and absorption. The research was developed from a survey of 78 companies in the automotive sector and data analysis was based on the statistical techniques of exploratory factor analysis, which combines measurable variables into latent factors, cluster analysis, which identifies clusters of companies, and discriminant analysis, which validates the statistical difference between the clusters identified. The results point to four different clusters regarding the practice of Knowledge Management: "Innovative Companies", "Explorative Companies", "Exploitative Companies" and "Companies Laggards".

Keywords: Cluster analysis. Factor analysis. Contextual factors. Knowledge management. Automotive industry.

\section{Introdução}

O conhecimento organizacional, no contexto atual das organizações, é considerado um ativo que, embora intangível, gera a elas vantagem competitiva. Esta é alcançada por meio do aperfeiçoamento contínuo e da inovação do processo produtivo e do produto, e o conhecimento é o recurso organizacional que permite à organização desenvolver tais atividades de melhoria e inovação (Grant, 1996; Torugsa; O'Donohue, 2016).

A missão principal da Gestão do Conhecimento (GC) é promover um processo contínuo de aquisição, armazenamento, distribuição e utilização de conhecimento (Chen; Huang; Siao, 2010; Gonzalez; Martins, 2014). A aquisição do conhecimento é o ponto de partida do processo de GC, manifestando-se pela capacidade de aprendizagem de seus indivíduos ou pela integração de conhecimento de origem externa (López-Sáez et al., 2010; White; Cicmil, 2016). Já a retenção do conhecimento consiste na capacidade da organização se apropriar e institucionalizar o conhecimento adquirido (Lin; Chang; Tsai, 2016). Por sua vez, a distribuição do conhecimento institucionalizado permite que os indivíduos da organização tenham acesso a uma base comum de conhecimento, promovendo sua utilização em novos processos e, consequentemente, sua transformação (Lefebvre et al., 2016).

A capacidade das organizações promoverem esse ciclo está relacionada com o desenvolvimento de fatores contextuais que o favoreçam. Esses fatores estabelecem o comportamento organizacional, no que se refere aos valores e crenças que guiam os indivíduos, à integração e forma de organização dos funcionários em grupos, ao nível de capacitação dos funcionários e à postura assumida pela direção da empresa. Sem o empenho quanto ao desenvolvimento desses fatores, quaisquer iniciativas organizacionais voltadas para a GC acabam não resultando nos benefícios esperados (Gonzalez; Martins, 2014).

Tendo em vista que esses fatores são desenvolvidos internamente e de forma bastante particular pelas organizações, e ainda que eles influenciam de forma direta o processo de GC, pode-se afirmar que as organizações possuem, consequentemente, maneiras e capacidades distintas de construir capital intelectual e obter aumento da capacidade inovativa (Chen; Huang; Siao, 2010; Gonzalez; Martins; Toledo, 2014; Torugsa; O'Donohue, 2016). Dessa forma, o objetivo principal deste artigo é estabelecer uma tipologia que caracterize grupos distintos em relação à prática da GC e determinar as implicações dessas características quanto à utilização do conhecimento em atividades que gerem vantagem competitiva em empresas do setor automobilístico.

A escolha desse setor pode ser justificada em dois sentidos. O primeiro refere-se à sua importância dentro da indústria brasileira. Segundo a Associação Nacional dos Fabricantes de Veículos Automotores (2016), esse segmento emprega 5,6\% dos funcionários da indústria brasileira e é responsável por 19,8\% do Produto Interno Bruto (PIB) industrial. Outra justificativa refere-se à intensidade de avanços tecnológicos que predomina nessa indústria (United Nations Conference on Trade and Development, 2005). Gonzalez e Martins (2014) caracteriza a indústria automobilística como um setor que apresenta um elevado grau de barreiras de entrada originadas do conhecimento, uma elevada persistência quanto à prática de inovação tecnológica, uma base de conhecimento complexa e, também, elevados graus de cumulatividade e apropriabilidade, que exige um sofisticado processo de GC. 
Autores como Yang (2010) tratam a GC como um processo com fases específicas, as quais têm como objetivo central a disseminação do conhecimento para sua posterior reutilização por outros indivíduos e grupos e consequente transformação de seu conteúdo, gerando novos conhecimentos. O sucesso da implementação desse processo é condicionado ao desenvolvimento de características organizacionais adequadas que propiciem o desenvolvimento de quatro fases: aquisição, armazenamento, distribuição e utilização do conhecimento (Liao; Chuang; To, 2011; Gonzalez; Martins; Toledo, 2014).

A aquisição refere-se ao processo intraorganizacional que facilita a criação de conhecimento tácito e explícito, partindo dos indivíduos e integrando-se ao nível organizacional, bem como a identificação e absorção de informação e conhecimento de origem externa (López-Saez et al., 2010). O processo de aquisição ainda está relacionado com o estímulo organizacional em torno da aprendizagem dos indivíduos, que torne a firma apta em integrar, construir e reconfigurar suas competências internas a fim de responder às mudanças ambientais (Cohen; Levinthal, 1990; Teece, 2007; Patterson; Ambrosini, 2015), por meio do desenvolvimento de uma cultura voltada à aprendizagem (Irani; Sharif; Love, 2009; Corfield; Paton, 2016; Marouf, 2016).

Já o estágio de armazenamento do conhecimento refere-se ao processo de formação de memória organizacional, na qual o conhecimento é formalmente armazenado em sistemas físicos de memória, relacionados a Sistemas de Informação (SI). Trata-se de um conhecimento informalmente retido na forma de valores, normas e crenças, que se associam à cultura e estrutura organizacional (Martins; Meyer, 2012; Corfiel; Paton, 2016), e, também, retido nos processos, ferramentas e rotinas organizacionais (Kane; Alavi, 2007). A firma, nesse contexto, passa a ter conotação de repositório de conhecimento (Grant, 1996), caracterizando-se como um local físico que sustenta a criação e desenvolvimento, provendo um contexto social.

Por sua vez, a distribuição do conhecimento diz respeito ao processo pelo qual novas informações de diferentes origens são compartilhadas e, eventualmente, podem dirigir a criação de novo conhecimento, entendimento e informação (Lefebvre et al., 2016; Van Dijk; Hendriks; Romo-Leroux, 2016). Os trabalhos mais antigos acerca do processo de transferência de conhecimento tinham como ênfase fatores cognitivos e sociais (Hwang; Shin; Argote, 2015). Atualmente, o foco incide sobre os fatores organizacionais que facilitam ou inibem o processo de transferência, incluindo a capacidade absortiva da organização (Lin; Chang; Tsai, 2016; Van Dijk; Hendriks; Romo-Leroux, 2016), o desenvolvimento de uma cultura de compartilhamento (Hwang; Shin; Argote, 2015; Van Dijk; Hendrik; Romo-Leroux, 2016), a expertise desenvolvida pelos indivíduos (Cross; Sproull, 2004), os aspectos motivacionais (Quigley et al., 2007) e a tecnologia, como fatores que facilitam o processo de transferência (Kane; Alavi, 2007).

Em relação à fase de utilização do conhecimento, Ganzaroli et al. (2016) a define como a habilidade dos indivíduos de uma organização em localizar, acessar e utilizar informação e conhecimento armazenados nos sistemas de memória formal e informal da organização. O conhecimento deve ser utilizado como base para o desenvolvimento de novos conhecimentos por meio da integração, inovação, criação e extensão da base de conhecimento existente, e ainda deve ser usado como base para a tomada de decisões. Desse modo, a utilização do conhecimento pode assumir seja um caráter explotativo, quando a partir dele são tomadas decisões ou melhorias, utilizando-se a mesma base do conhecimento; seja um caráter explorativo, quando essa base é utilizada como conhecimento primário para a criação de novos conhecimentos, numa proposta inovativa (Cohen; Levinthal, 1990; Rowley, 2001).

Para Magnier-Watanable e Senoo (2008), a forma de utilização do conhecimento - explotativa ou explorativa -, está diretamente relacionada à estratégia escolhida pela empresa: reativa ou inovativa. A primeira estratégia explota o conhecimento existente, isto é, limita-se a utilizá-lo a fim de viabilizar uma estratégia definida. Por outro lado, a estratégia inovativa usa um modelo explorativo a fim de competir de forma diferente no mercado atual ou em novos mercados. 


\section{Constructos organizacionais que promovem a gestão do conhecimento}

Esta pesquisa parte da premissa de que a GC é um fenômeno técnico-social (Lin, 2007; Van Dijk; Hendriks; Romo-Leroux, 2016) e, dessa forma, as etapas do processo de GC estão condicionadas ao desenvolvimento organizacional. Seguindo pesquisas prévias realizadas por Liao, Chuang eTo (2011), Martins e Meyer (2012) e Gonzalez e Martins (2014) o êxito do processo de GC depende da implementação de cinco constructos organizacionais: Desenvolvimento de recursos humanos; Trabalho em equipe; Cultura organizacional; estrutura organizacional; e desenvolvimento e absorção de conhecimento. Os autores salientam que esses constructos contemplam as características necessárias às empresas para caminharem no sentido de uma organização de aprendizagem, isto é, cujo conhecimento flui do nível individual para o grupal, até ser institucionalizado no nível organizacional (Cross; Sproull, 2004).

Em relação ao desenvolvimento de recursos humanos, Gonzalez e Martins (2014) enfatizam que nenhuma organização pode gerar conhecimento sem pessoas habilitadas. As abordagens mais modernas relacionadas ao tratamento dos recursos humanos partem de premissas voltadas ao desenvolvimento da mão de obra, visando ao aprimoramento constante das competências (Zangiski; Lima; Costa, 2013). E ainda, por meio das práticas de desenvolvimento de recursos humanos, as organizações podem desenvolver uma cultura organizacional que encoraje a aquisição e o compartilhamento do conhecimento (Zangiski; Lima; Costa, 2013).

O trabalho em equipe - segundo constructo considerado nesta pesquisa - é uma das principais características das formas mais modernas de organização do trabalho, como a manufatura enxuta e os grupos semiautônomos (Lee; To; Ty, 2013; Gonzalez; Martins, 2014). Existem diferentes projetos de trabalho em grupo: por exemplo, enquanto na manufatura enxuta, o conceito de grupo é focado na utilização de procedimentos operacionais e hierarquia bem definida com supervisão rígida, já nos grupos com base num modelo sociotécnico existe certo grau de autonomia quanto à escolha do melhor método de trabalho, bem como uma hierarquia mais enxuta (Lee; To; Ty, 2013).

A visão da 'organização como máquina' torna-se cada vez mais ultrapassada quando se visa estudar uma forma de gestão que propicie a constante aquisição e distribuição do conhecimento, ou seja, um fluxo intenso de conhecimento (Hwang; Sinh; Argote, 2015). Em razão dessa prerrogativa, a GC depende de um contexto social alicerçado por trabalho em equipe - grupos que compartilham ideias e competências profissionais (Hwang; Sinh; Argote, 2015) -, de modo a promover a aprendizagem contínua dos indivíduos. Um aspecto central para a efetividade do trabalho em grupo é o processo de integração do conhecimento (Lee; To; Ty, 2013). Para que se torne útil, o conhecimento individual, que é propriedade dos indivíduos, deve ser integrado aos grupos por meio de um processo de assimilação e interpretação e institucionalizado na organização (Grant, 1996) para que possa ser explotado a fim de gerar vantagem competitiva para ela.

Quanto à cultura organizacional - terceiro constructo desta pesquisa -, a literatura existente em GC salienta constantemente a inseparável relação entre cultura organizacional e GC (Van Dijk; Hendriks; Romo-Leroux, 2016; Marouf, 2016). A pesquisa realizada por Marouf (2016) aponta que, quando uma organização adota um sistema de gerenciamento do conhecimento sem se preocupar com o desenvolvimento cultural que o propicie, a eficiência da GC é limitada. Assim, cultura organizacional é o terceiro constructo que esta pesquisa considera.

Corfield e Paton (2016) definem a cultura do conhecimento como os pressupostos estabelecidos pela organização que valorizam o compartilhamento e integração do conhecimento entre indivíduos e grupos. Em relação à questão do compartilhamento do conhecimento, Gonzalez e Martins (2014) concluem que as organizações com valores mais abertos, e voltadas para o apoio recíproco entre os indivíduos, são predispostas a construir uma cultura do conhecimento.

Tomada aqui como quarto constructo deste estudo, a estrutura organizacional estabelece o modelo como as empresas se organizam e o grau com que o conhecimento circula dentro delas e entre elas. Segundo Gonzalez, 
Martins e Toledo (2014), as firmas devem adotar uma estrutura organizacional que permita criar e transferir conhecimento tanto quanto possível. Os autores recomendam a adoção de estruturas que não "sufoquem" a organização em relação ao fluxo de conhecimento. Tendo em vista a relação íntima entre a estrutura organizacional e o processo de disseminação e integração do conhecimento, ela foi utilizada como constructo nesta pesquisa.

Chen e Huang (2007) definem estrutura organizacional a partir de três elementos: a formalização, a centralização e a integração. A formalização refere-se ao nível de codificação de regras e procedimentos que guiam o comportamento dos trabalhadores. Em organizações altamente formalizadas, a existência de documentos formais inibe a geração de ideias e impede o comportamento espontâneo necessário ao estímulo às inovações (Massaro et al., 2016). Já a centralização está relacionada à concentração do poder decisório aos altos níveis hierárquicos (Gonzalez; Martins; Toledo, 2014; Massaro et al., 2016). Por fim, a integração refere-se ao grau de inter-relacionamento entre indivíduos e setores da organização (Liao; Chuang; To, 2011; Lefebvre et al., 2016). Destaca-se o papel dos sistemas de informação como mecanismo de suporte aos processos de formalização e disseminação do conhecimento formalizado (Martins; Meyer, 2012).

Finalmente, a capacidade de desenvolvimento e absorção de conhecimento - quinto constructo considerado nesta pesquisa -, refere-se à aptidão das organizações em desenvolver e absorver conhecimento. A capacidade de absorção refere-se à habilidade de uma organização em reconhecer o valor de determinado conhecimento, assimilá-lo e aplicá-lo, visando obter vantagem competitiva (Cohen; Levinthal, 1990; Lin; Chang; Tsai, 2016). A noção fundamental desse conceito concentra-se no fato de que as organizações precisam acessar seu conhecimento primário para assimilar e utilizar novos conhecimentos, isto é, o acúmulo de conhecimento primário aumenta o potencial de aprendizagem futura (Garicano; Wu, 2012).

Organizações com maior nível de capacidade de absorção tendem a ser mais dinâmicas (Lin; Chang; Tsai, 2016), isto é, aptas a explorar oportunidades no ambiente, independentemente da performance atual, enquanto as organizações com menor nível de capacidade de absorção tendem a ser mais reativas, pois procuram formas para a correção de suas falhas, baseando-se em padrões de desempenho que não significam avanço tecnológico. Os conceitos de reatividade e proatividade organizacional são de longo prazo, isto é, as firmas que atingem um nível proativo, por exemplo, permanecem dessa forma pela sua própria aspiração em pesquisar novas oportunidades (Cohen; Levinthal, 1990, Garicano; Wu, 2012).

Exploração e explotação representam dois modelos fundamentalmente diferentes para a aprendizagem organizacional. O primeiro implica um comportamento voltado à pesquisa, descoberta e experimentação, enquanto o segundo caracteriza-se pelo refinamento, implementação, eficiência, produção e seleção (March, 1991; Ganzaroli et al., 2016). March (1991) enfatiza que os retornos associados à exploração são mais variáveis e de longo prazo, enquanto os retornos relacionados à explotação são mais precisos e de curto prazo.

\section{Procedimentos metodológicos}

Forza (2002) indica que, após a definição do objetivo de pesquisa, a próxima etapa consiste em levantar o referencial teórico que oriente a determinação das variáveis e, em seguida, definir a amostra a ser pesquisada. Seguindo tais parâmetros, procedeu-se à revisão bibliográfica dos artigos presentes nas principais revistas que tratam do tema GC. A seleção das revistas levou em conta dois fatores: índice JCR superior a 1,0 e temática referente a informação e conhecimento e ciências gerenciais. A pesquisa foi centrada nas bases Elsevier, Emerald, Informs PubsOnline, Taylor and Francis e Inderscience, tendo como principais revistas pesquisadas "Journal of Knowledge Management", "Organization Science", "International Journal of Production Economics", "International Journal of Information Management", "Strategic Management Journal" e "Journal of Business Research". 
Como palavras-chave, foram buscados os termos "gestão do conhecimento", associado a "cultura organizacional", "recursos humanos", "trabalho em grupo", "estrutura organizacional", "absorção de conhecimento", "aquisição de conhecimento", "armazenamento de conhecimento", "distribuição de conhecimento" e "utilização de conhecimento".

Em relação ao estabelecimento da amostra, ela foi concebida a partir da lista de empresas cadastradas no catálogo do Sindipeças (Sindicato Nacional da Indústria de Componentes para Veículos Automotores) no site $<$ http://www.sindipecas.org.br>. Em relação ao funcionário pesquisado, foram considerados os cargos de direção, gerência e supervisão dos departamentos de recursos humanos, produção, qualidade e projetos.

O questionário de pesquisa foi constituído de duas partes. A primeira trata de questões que caracterizam a empresa e o funcionário; e a segunda refere-se aos cinco constructos descritos abaixo:

1) Variáveis do constructo "Desenvolvimento dos Recursos Humanos":

- RH1: A empresa possui um método estruturado para a avaliação das competências exigidas dos funcionários.

- $\mathrm{RH2}$ : Os gestores acompanham e avaliam constantemente o desenvolvimento de seus subordinados.

- RH3: A empresa oferece cursos/palestras aos funcionários para o aprimoramento das competências.

- RH4: Os profissionais da empresa buscam o autodesenvolvimento.

- RH5: Os profissionais da empresa estão capacitados a tomar decisões e gerenciar riscos.

- RH6: A empresa apresenta mecanismos de premiação e recompensas por resultados atingidos.

- RH7: Os funcionários reconhecem a importância de seus trabalhos em relação à estratégia da empresa.

- RH8: O processo de recrutamento valoriza a postura criativa e o espírito empreendedor.

- RH9: Os funcionários são altamente polivalentes, isto é, exercem diversas funções relativas aos seus processos de trabalho.

2) Variáveis do constructo "Trabalho em Equipe":

- TE1: Os projetos de trabalho são realizados, em sua maioria, em grupos que executam e analisam os resultados conjuntamente.

- TE2: A empresa incentiva o aprendizado e a troca de informações entre os funcionários.

- TE3: A empresa oferece aos funcionários autonomia para resolverem problemas operacionais.

- TE4: A organização estimula a interação entre funcionários de diferentes especialidades.

- TE5: Os funcionários de um determinado setor ou grupo criam uma linguagem comum entre eles, relativa ao processo de trabalho.

- TE6: Os funcionários dos grupos de trabalho têm a capacidade de se autogerenciarem, isto é, capacidade de auto-organização.

- TE7: É perceptível que os funcionários de um determinado setor ou grupo de trabalho se sentem "donos" daquela área.

- TE8: Os funcionários de um grupo têm ciência dos conhecimentos e habilidades dominados por seus colegas de trabalho.

3) Variáveis do constructo "Cultura Organizacional"

- CO1: Os funcionários costumam trocar informações e experiências quando se deparam com um problema.

- CO2: Após sessões de treinamento, os funcionários interagem sobre os conhecimentos adquiridos. 
- CO3: Os funcionários estão cientes quanto à evolução da empresa e de suas necessidades individuais de qualificação.

- CO4: Quando um funcionário apresenta uma ideia ou projeto de melhoria, existe a cooperação dos demais colegas.

- CO5: Quando um funcionário apresenta uma ideia ou projeto de melhoria, existe o reconhecimento de seu superior.

- CO6: Existe uma comunicação constante entre funcionários e gerência sobre a situação da empresa.

- CO7: A empresa apresenta uma cultura que estimula seus funcionários a tomarem decisões e assumirem riscos inerentes aos seus processos.

- CO8: A empresa interpreta eventuais erros, cometidos pelos funcionários, como parte do processo de aprendizagem.

- CO9: Os gestores da empresa conseguem identificar as melhores práticas dos processos pelos quais são responsáveis.

4) Variáveis do constructo "Estrutura Organizacional":

- EO1: A empresa possui poucos níveis hierárquicos, isto é, ela apresenta uma estrutura verticalmente enxuta.

- EO2: Existe facilidade de comunicação entre os setores ou departamentos da empresa.

- EO3: Existe um grande interesse da organização em estabelecer procedimentos, métodos ou instruções para as atividades.

- EO4: Sua empresa consegue mudar a estrutura de departamentos, cargos e atribuições quando necessário.

- EO5: É comum diversos projetos ou atividades envolverem dois ou mais setores da empresa, simultaneamente.

- EO6: Os sistemas de informação da sua empresa estão integrados, sendo possível que qualquer área da empresa tenha acesso ao conteúdo das demais.

- EO7: As melhores práticas de cada departamento são registradas em um banco de dados.

- EO8: Existe amplo acesso ao banco de dados da empresa, independentemente do nível hierárquico do funcionário.

- EO9: A empresa apresenta ambientes virtuais para discussão entre os funcionários (fóruns virtuais, grupos de e-mails, MSN, entre outros).

5) Variáveis do constructo "Desenvolvimento e Absorção de Conhecimento":

- CAbs1: A sua empresa desenvolve novas tecnologias internamente.

- CAbs2: O conhecimento desenvolvido pelos funcionários é utilizado em atividades de melhoria.

- CAbs3: A utilização do conhecimento e competências, adquiridos durante o tempo, mantém sua empresa competitiva frente aos concorrentes.

- CAbs4: Sua empresa tem fácil acesso a novas tecnologias (originados de especialistas externos, de universidades, parceria com outras empresas etc.).

- CAbs5: Sua empresa tem facilidade em aplicar novas tecnologias e conhecimentos.

- CAbs6: Os processos e atividade praticados rotineiramente evoluem com o tempo. 
- CAbs7: Sua empresa domina a tecnologia envolvida em sua área de atuação.

- CAbs8: Sua empresa antecipa inovações no mercado em que concorre.

- CAbs9: As competências desenvolvidas internamentefacilitam à sua empresa explorar novas oportunidades no mercado.

- CAbs10: Sua empresa possui um método estruturado para solução de problema (procedimento, utilização de ferramentas, formação de equipes etc.).

- CAbs11: Sua empresa tem fácil acesso a novas tecnologias (originadas de especialistas externos, universidades, parceria com outras empresas etc).

As questões da segunda parte foram medidas a partir de uma escala Likert de seis pontos, em que 1 significa "nunca praticado ou observado", e 6 significa "faz parte da rotina da empresa".

Em seguida, o questionário validado foi enviado para 450 empresas, escolhidas aleatoriamente do banco de dados do Sindipeças, via e-mail, juntamente com uma carta de apresentação da pesquisa, no período de novembro de 2015 a março de 2016. Para aumentar a taxa de retorno, antes do envio do e-mail, o pesquisador realizou um contato telefônico com a empresa a fim de elucidar a importância do estudo. A taxa de retorno obtida foi de 88 questionários (19,5\%), dos quais 10 foram descartados por falha de preenchimento, totalizando 78 questionários válidos (17,3\%). Cada empresa apresentou apenas um respondente com cargo de nível de direção ou gerência na área de engenharia, qualidade ou recursos humanos.

Segundo Mingoti (2005), em pesquisas na área de ciências sociais aplicadas, uma condição de $(n-p)>30$, onde $n$ representa o número de observações e p representa o número de variáveis, apresenta-se como satisfatória. No presente estudo, tem-se $n=78$ e $p=46$, e, portanto, satisfazendo a condição prescrita pelo autor.

Preacher e MacCallum (2002) ressaltam que as técnicas multivariadas de dados, como a análise fatorial e análise de clusters, podem alcançar bons resultados com amostras relativamente pequenas. Tanto Preacher e MacCallum (2002) quanto Costello e Osborne (2005), enfatizam que a melhor de forma de se evidenciarem "dados fortes" (Costello; Osborne, 2005) em análise multivariada se dá por meio de três constatações. A primeira relacionase às cargas elevadas das variáveis em relação aos fatores, superiores a 0,50; a segunda refere-se a um número elevado de variáveis carregando fortemente um mesmo fator, com pelo menos três variáveis por fator; e a terceira diz respeito à ausência de cargas fatoriais cruzadas, isto é, uma mesma variável relacionando-se fortemente com dois ou mais fatores simultaneamente (Preacher; MacCallum, 2002; Costello; Osborne, 2005).

Os dados coletados foram compilados em uma planilha de Excel. O software SPSS (Statistical Package for the Social Sciences) versão 21.0 foi utilizado para analisar os dados. Inicialmente, foram testados os dados de confiabilidade ( $\alpha$ de Cronbach) e validade, medida por meio da carga fatorial, obtida a partir do procedimento de análise de componente principal. Segundo Preacher e MacCallum (2002), Hair Jr. et al. (2005), e Costello e Osborne (2005), o $\alpha$ de Cronbach deve ser superior a 0,700 e a carga fatorial das variáveis de pesquisa deve ser superior a 0,500. Os valores de $\alpha$ de Cronbach foi de 0,783 para o fator Desenvolvimento de Recursos Humanos; 0,761 para o constructo Trabalho em Equipe; 0,822 para Cultura Organizacional; 0,864 para Estrutura Organizacional; e 0,802 para Desenvolvimento e Absorção de Conhecimento. Em relação às cargas fatoriais, os valores obtidos compreenderam-se no intervalo entre 0.685 e 0.9106 para as 46 variáveis medidas. Assim, todas as variáveis e fatores obtiveram valores satisfatórios quanto à confiabilidade e validade (Hair Jr. et al., 2005; Costello; Osborne, 2005).

A primeira análise multivariada desenvolvida foi a fatorial, que constitui uma técnica estatística exploratória, destinada a resumir um conjunto de variáveis em um conjunto de fatores não observáveis, denominado Análise Fatorial Tipo R (Hair Jr. et al., 2005). Na presente pesquisa, a análise fatorial permite condensar um conjunto grande de variáveis, composto originalmente por 46 delas, em conjunto reduzido de fatores que permite a identificação 
e melhor caracterização daqueles que sustentam o processo de GC. O resultado da análise fatorial, detalhado na próxima seção, mostra a formação de oito fatores, todos associados fortemente com pelo menos três variáveis. Evidencia-se ainda o baixo carregamento cruzado das variáveis em relação aos fatores. Essas considerações, em linha com Preacher e MacCallum (2002) e Costello e Osborne (2005), permitem concluir que os dados são adequados e confiáveis à condução da pesquisa.

A análise de clusters realizada nesta pesquisa tem por objetivo desenvolver uma tipologia, baseada em dados empíricos, identificando similaridades entre os casos em relação às variáveis levantadas teoricamente e, consequentemente, simplificando a análise dos casos por meio do agrupamento. A tipologia analisada neste trabalho consiste na classificação de agrupamentos de empresas, levando-se em consideração o desenvolvimento de fatores contextuais da organização nas indústrias pesquisadas, utilizando-se, para tanto, a estrutura de fatores estabelecida pela análise fatorial.

\section{Resultados}

\section{Caracterização das empresas e entrevistados pesquisados}

A primeira análise realizada visa compreender o porte das empresas pesquisadas. Todas as empresas consideradas pela pesquisa são de médio ou grande porte, sendo que 46\% possuem entre 500 e 5 mil funcionários, 41\% possuem entre 100 e 500 funcionários, e 13\% possuem mais que 5 mil funcionários.

Em relação ao tempo de atuação no mercado automobilístico, foram pesquisadas organizações com pelo menos cinco anos no mercado. Os dados apontam que a maior concentração foi de empresas com tempo de atuação entre dez e vinte anos (33,0\%) e com mais de quarenta anos no mercado (32,0\%). O número de empresas com tempo entre cinco e dez anos foi pequeno (7,7\%).

O estudo de fatores contextuais da organização exige que o entrevistado possua uma visão mais aprofundada do contexto organizacional. Assim, foram considerados os níveis hierárquicos de direção, gerência e supervisão, os quais possuem poder de decisão. Os dados coletados apontam que a maioria dos respondentes é de nível gerencial (53\%), enquanto 25\% são supervisores e 22\% são diretores nas funções consideradas pela pesquisa.

\section{Análise multivariada dos dados}

Inicialmente, para análise dos dados, foram verificados os pressupostos estatísticos de consistência interna da escala das variáveis observadas e dos dados coletados. Quanto à consistência da escala, foi utilizado o coeficiente a de Cronbach, enquanto a análise de adequação dos dados foi realizada por meio dos testes de esfericidade de Bartlett (BTS) e teste KMO.

Os dados coletados apresentaram um valor satisfatório quanto à adequação da amostra (KMO=0.867), pois estão acima de 0,50 (Hair Jr. et al., 2005) e a hipótese de matriz-identidade foi invalidada pelo teste de Bartlett a um nível de significância de 5\%. $O \alpha$ de Cronbach superior a 0,70 $(\alpha=0,782)$ também aponta que a escala apresenta confiabilidade (Hair Jr. et al., 2005).

Em seguida, foi observada a quantidade de fatores extraídos relevantes e seus respectivos poderes explanatórios. Conforme a Tabela 1, apenas oito fatores são mantidos no estudo, apresentando Eingenvalue superior a 1,0 e explicando 81,70\% da variância, acima do percentual recomendado por Hair Jr. et al. (2005) para pesquisas em ciências sociais. 
Tabela 1. Fatores extraídos e porcentagem da variância explicada.

\begin{tabular}{|c|c|c|c|c|c|c|c|c|c|}
\hline \multirow{2}{*}{ Componente } & \multicolumn{3}{|c|}{ Eingenvalue Inicial } & \multicolumn{3}{|c|}{$\begin{array}{l}\text { Soma dos quadrados dos fatores } \\
\text { extraídos }\end{array}$} & \multicolumn{3}{|c|}{$\begin{array}{c}\text { Soma dos quadrados dos fatores } \\
\text { rotacionados }\end{array}$} \\
\hline & Total & $\begin{array}{c}\text { \% da } \\
\text { variância }\end{array}$ & $\%$ Acumulada & Total & $\begin{array}{c}\text { \% da } \\
\text { variância }\end{array}$ & $\%$ Acumulada & Total & $\begin{array}{c}\text { \% da } \\
\text { variância }\end{array}$ & $\%$ Acumulada \\
\hline 1 & 24.367 & 52.971 & 52.791 & 24.367 & 52.971 & 52.791 & 6.790 & 14.760 & 14.760 \\
\hline 2 & 3.327 & 7.233 & 60.204 & 3.327 & 7.233 & 60.204 & 6.722 & 14.613 & 29.373 \\
\hline 3 & 2.386 & 5.186 & 65.590 & 2.386 & 5.186 & 65.590 & 6.303 & 13.703 & 43.076 \\
\hline 4 & 1.852 & 4.026 & 69.416 & 1.852 & 4.026 & 69.416 & 3.918 & 8.517 & 51.594 \\
\hline 5 & 1.711 & 3.721 & 73.136 & 1.711 & 3.721 & 73.136 & 3.736 & 8.122 & 59.715 \\
\hline 6 & 1.399 & 3.042 & 76.178 & 1.399 & 3.042 & 76.178 & 3.673 & 7.985 & 67.701 \\
\hline 7 & 1.350 & 2.936 & 79.114 & 1.350 & 2.936 & 79.114 & 3.381 & 7.350 & 75.051 \\
\hline 8 & 1.193 & 2.953 & 81.707 & 1.193 & 2.953 & 81.707 & 3.062 & 6.656 & 81.707 \\
\hline
\end{tabular}

Fonte: Elaborada pelos autores (2017).

A Tabela 2 apresenta a matriz de componentes rotacionados a partir do método Varimax ${ }^{4}$. A carga fatorial mínima para a manutenção de uma variável em um determinado fator, em função do tamanho da amostra da pesquisa, é de 0,5, portanto, evidencia-se que todas as variáveis são mantidas em seus respectivos fatores.

A etapa seguinte consiste em nomear os oito fatores retidos em função das variáveis que cada um deles absorveu. Os seguintes fatores foram identificados a partir das variáveis agrupadas na fase anterior:

1) Resolução de problemas e melhoria incremental.

2) Cultura de aprendizagem e trabalho em equipe.

3) Postura proativa e estrutura organizacional enxuta.

4) Conhecimento primário e identidade compartilhados.

5) Capacidade de absorção de conhecimento.

6) Mapeamento e avaliação e competências.

7) Estratégia inovadora.

8) Sistema de informação.

Para utilização dos dados da análise fatorial em etapas posteriores, é necessário determinar os escores fatoriais para cada empresa pesquisada. Os escores fatoriais substituem os dados originais coletados junto às empresas, sendo tal substituição devida ao fato de que a análise fatorial reduz os dados originais em fatores comuns. Dessa forma, os dados de entrada da análise de cluster são constituídos pelos escores fatoriais dos oito fatores retidos, referentes às 78 empresas pesquisadas.

O procedimento de agrupamento utilizado foi o método Ward (hierárquico). A cada estágio são aglomerados dois casos em razão de sua proximidade (baixa distância euclidiana). Dessa forma, cada novo estágio que a técnica propõe representa uma redução de um cluster. Deve-se reduzir a quantidade de clusters até o ponto que a variação do coeficiente de aglomeração não se eleve drasticamente em função dessa redução.

A partir dos valores de centroide, que são as médias dos oito escores fatoriais das empresas para cada um dos quatro clusters (Tabela 3), é possível caracterizar os agrupamentos. Deve-se salientar que os escores fatoriais são

${ }_{4}$ Varimax consiste em um método de rotação ortogonal, amplamente utilizado em análise fatorial, que resulta em componentes principais ou fatores compostos de variáveis com pesos significativos e as demais variáveis com pesos próximos a zero, oferecendo maior potencial de interpretabilidade dos fatores e tornando a solução fatorial mais simples e significativa (Hair Jr. et al., 2005). 
Tabela 2. Matriz de componentes rotacionada (Rotação Varimax).

\begin{tabular}{|c|c|c|c|c|c|c|c|c|}
\hline Variáveis & 1 & 2 & 3 & 4 & 5 & 6 & 7 & 8 \\
\hline $\mathrm{CO} 4$ & 0,7136 & 0,2726 & 0,1626 & 0,4182 & 0,0115 & 0,0770 & $-0,0159$ & 0,1708 \\
\hline $\mathrm{CO} 5$ & 0,7327 & 0,3363 & 0,2001 & 0,1366 & $-0,1170$ & 0,0487 & 0,0738 & 0,0899 \\
\hline TE3 & 0,7662 & 0,1897 & 0,2225 & 0,3080 & $-0,0189$ & $-0,0849$ & 0,1748 & 0,0379 \\
\hline EO5 & 0,7330 & 0,1673 & 0,3094 & $-0,0606$ & 0,0116 & 0,0906 & 0,2941 & 0,0435 \\
\hline CABS2 & 0,7337 & 0,1306 & 0,1828 & 0,0631 & 0,1866 & 0,3113 & 0,2081 & 0,2923 \\
\hline CABS3 & 0,7320 & 0,1524 & 0,2277 & 0,0552 & 0,1980 & 0,1930 & 0,3550 & 0,1265 \\
\hline CABS6 & 0,7498 & 0,2444 & 0,1996 & 0,0428 & 0,2066 & 0,1780 & 0,2039 & 0,2049 \\
\hline CABS10 & 0,7507 & 0,1617 & 0,2752 & 0,0455 & 0,2147 & 0,2159 & 0,1619 & 0,1630 \\
\hline $\mathrm{CO} 1$ & 0,2329 & 0,6137 & 0,0829 & 0,4876 & 0,2152 & 0,1239 & 0,2869 & 0,1706 \\
\hline $\mathrm{CO} 2$ & 0,2707 & 0,5582 & 0,1621 & 0,3447 & 0,2417 & 0,2856 & 0,1905 & 0,2728 \\
\hline CO6 & 0,2173 & 0,7934 & 0,2249 & 0,1844 & 0,0926 & 0,2185 & 0,2262 & 0,1471 \\
\hline $\mathrm{CO} 7$ & 0,2799 & 0,7413 & 0,2500 & 0,2381 & 0,2272 & 0,1966 & 0,0270 & 0,2693 \\
\hline $\mathrm{CO} 8$ & 0,2942 & 0,6576 & 0,2543 & 0,1485 & 0,3341 & 0,1528 & 0,1012 & 0,1594 \\
\hline co9 & 0,2573 & 0,7302 & 0,1438 & 0,3231 & 0,1847 & 0,1836 & 0,2121 & 0,2666 \\
\hline TE1 & 0,2519 & 0,7050 & 0,3116 & 0,0828 & 0,2307 & 0,2959 & 0,1788 & 0,0985 \\
\hline ET2 & 0,3313 & 0,6874 & 0,2200 & 0,1543 & 0,3315 & 0,2181 & 0,2052 & 0,1600 \\
\hline TE4 & 0,2386 & 0,6934 & 0,2996 & 0,2397 & 0,1963 & 0,2228 & 0,2140 & 0,0695 \\
\hline $\mathrm{RH} 4$ & 0,2758 & 0,0946 & 0,7079 & 0,2153 & 0,1988 & 0,2111 & 0,2012 & 0,1467 \\
\hline $\mathrm{RH} 5$ & 0,2620 & 0,1576 & 0,6541 & 0,2374 & 0,2648 & 0,1518 & 0,1841 & 0,1569 \\
\hline $\mathrm{RH} 6$ & 0,3764 & 0,2537 & 0,6558 & 0,1365 & 0,2643 & 0,1972 & $-0,0737$ & 0,0957 \\
\hline $\mathrm{RH} 7$ & 0,2864 & 0,2484 & 0,5987 & 0,2423 & 0,4040 & $-0,0284$ & 0,1500 & 0,1340 \\
\hline $\mathrm{RH} 8$ & 0,3573 & 0,1387 & 0,6709 & 0,2097 & 0,2330 & 0,3032 & 0,0315 & 0,2210 \\
\hline $\mathrm{RH} 9$ & 0,1394 & 0,2490 & 0,7955 & 0,1530 & 0,1705 & $-0,0768$ & $-0,0549$ & 0,0114 \\
\hline $\mathrm{CO} 3$ & 0,4333 & 0,2295 & 0,6590 & 0,0608 & 0,1812 & 0,1921 & 0,0864 & 0,2157 \\
\hline EO1 & 0,0966 & 0,2057 & 0,6050 & 0,2190 & $-0,1471$ & 0,1337 & 0,5072 & 0,2248 \\
\hline EO2 & 0,1730 & 0,2304 & 0,7221 & 0,1404 & $-0,1406$ & 0,1447 & 0,3564 & 0,1435 \\
\hline EO4 & 0,2995 & 0,0726 & 0,5637 & 0,2486 & $-0,1586$ & 0,2972 & 0,4028 & 0,1966 \\
\hline TE5 & 0,1933 & 0,3519 & 0,3737 & 0,6288 & 0,0917 & 0,2246 & 0,1525 & $-0,1149$ \\
\hline TE6 & 0,2230 & 0,2512 & 0,2512 & 0,7147 & 0,2500 & 0,2003 & 0,0615 & 0,2561 \\
\hline TE7 & 0,0849 & 0,3209 & 0,3572 & 0,7374 & 0,0703 & 0,1628 & $-0,0049$ & 0,0496 \\
\hline TE8 & 0,1242 & 0,1886 & 0,2246 & 0,7731 & 0,2719 & 0,1973 & 0,1524 & 0,2747 \\
\hline CABS1 & 0,1568 & 0,3929 & 0,1766 & 0,1734 & 0,7246 & 0,1971 & 0,2402 & 0,1457 \\
\hline CABS4 & 0,1291 & 0,3726 & 0,2534 & 0,1683 & 0,6361 & 0,3209 & 0,1697 & 0,2859 \\
\hline CABS5 & 0,0052 & 0,4072 & 0,3490 & 0,1639 & 0,5989 & 0,2743 & 0,1735 & 0,1778 \\
\hline CABS11 & 0,0800 & 0,2680 & 0,1159 & 0,2170 & 0,7795 & 0,2180 & 0,1144 & 0,1298 \\
\hline $\mathrm{RH} 1$ & 0,1986 & 0,2902 & 0,2724 & 0,1046 & 0,2284 & 0,7871 & 0,0736 & 0,0583 \\
\hline $\mathrm{RH} 2$ & 0,2183 & 0,2562 & 0,1817 & 0,1717 & 0,1588 & 0,7642 & $-0,0408$ & 0,1651 \\
\hline $\mathrm{RH} 3$ & 0,1705 & 0,3392 & 0,0380 & 0,1950 & 0,1297 & 0,6412 & 0,2284 & 0,1466 \\
\hline EO3 & $-0,0029$ & 0,2368 & 0,1622 & 0,4400 & 0,2587 & 0,6052 & 0,1761 & 0,1088 \\
\hline CABS7 & 0,3897 & 0,2575 & 0,1611 & 0,0365 & 0,1436 & 0,0385 & 0,7659 & $-0,0300$ \\
\hline CABS8 & 0,3415 & 0,2447 & 0,0744 & 0,1319 & 0,3361 & 0,1135 & 0,7142 & 0,1068 \\
\hline CABS9 & 0,3239 & 0,2485 & 0,2304 & 0,1015 & 0,2437 & 0,0915 & 0,7321 & 0,1583 \\
\hline EO6 & 0,1132 & 0,1838 & 0,2515 & 0,1427 & 0,1198 & 0,0527 & 0,3309 & 0,7207 \\
\hline EO7 & 0,3522 & 0,4015 & 0,1764 & 0,2123 & 0,1294 & 0,2496 & $-0,0037$ & 0,5762 \\
\hline EO8 & 0,2268 & 0,3143 & 0,2193 & 0,0884 & 0,1490 & 0,0725 & 0,0349 & 0,7893 \\
\hline EO9 & 0,3400 & 0,0888 & 0,1044 & 0,1351 & 0,2609 & 0,3849 & $-0,0417$ & 0,5954 \\
\hline
\end{tabular}

Nota: RH: Recursos Humanos; CO: Cultura Organizacional; EO: Estrutura Organizacional; CABS: Desenvolvimento e Abstração do Conhecimento; TE: Trabalho em Equipe. Fonte: Elaborada pelos autores (2017). 
Tabela 3. Médias dos escores fatoriais para cada cluster.

\begin{tabular}{|c|c|c|c|c|c|c|c|c|}
\hline \multirow{2}{*}{ Clusters } & \multicolumn{8}{|c|}{ Fatores } \\
\hline & 1 & 2 & 3 & 4 & 5 & 6 & 7 & 8 \\
\hline 1 & 0,1777 & $-0,1058$ & 0,2523 & 0,0062 & $-0,3835$ & $-0,6788$ & $-0,1313$ & $-0,1228$ \\
\hline 2 & $-0,0896$ & 1,0788 & $-0,1991$ & 0,9320 & 0,3277 & 0,5528 & 0,8947 & 0,2582 \\
\hline 3 & $-0,0301$ & $-0,0715$ & 0,3450 & $-0,1604$ & 0,2344 & 0,4638 & 0,0102 & 0,1814 \\
\hline 4 & $-0,1562$ & $-1,1335$ & $-1,5778$ & $-0,8338$ & $-0,4054$ & $-0,0798$ & $-1,1058$ & $-0,2677$ \\
\hline
\end{tabular}

Fonte: Elaborada pelos autores (2017).

valores padronizados em z, e, portanto, não podem ser comparados às notas de 1 a 6 que foram atribuídas pelos respondentes por meio do questionário. Os valores padronizados são utilizados para a comparação dos clusters.

O cluster 1 aglomera 32 casos (41\%) e apresenta a maior valorização em relação ao fator Resolução de problemas e melhoria incremental e Postura proativa e estrutura organizacional enxuta. Esse grupo apresenta pouco destaque em relação aos fatores "Capacidade de absorção de conhecimento", "Estratégia inovadora" e "Mapeamento, Avaliação e Desenvolvimento de competências". Por tais características esse cluster é nomeado de "Empresas explotatoras".

O cluster 2, composto de 12 empresas (15,4\%), apresenta centroides relevantes em todos os fatores, tendo, inclusive, apresentado centroide significativo em relação à estratégia inovadora. Frente a tais características, este cluster é identificado como "Empresas inovadoras".

O cluster 3, composto de 25 empresas (32,1\%), apresenta centroides moderados em todos os fatores e centroides relativamente elevados em relação aos fatores "Capacidade de absorção de conhecimento," "Postura proativa e Estrutura organizacional enxuta" e "Mapeamento, Avaliação e Desenvolvimento de competências". Contudo, esse cluster não apresenta grande vocação à inovação e, dessa forma, os integrantes desse grupo foram classificados como "Empresas exploradoras de conhecimento".

O cluster 4, formado por 9 empresas (11,5\%), é aquele que apresenta o pior desempenho em relação aos fatores pesquisados. Nenhum dos oito fatores obteve centroide padronizado positivo. Os dois destaques do grupo são os fatores "Resolução de problemas e melhoria incremental" e "Mapeamento, Avaliação e Desenvolvimento de competências", de forma bastante modesta comparativamente aos demais agrupamentos. Dessa forma, os integrantes deste cluster são classificados como "Empresas retardatárias".

A Tabela 4 apresenta os dados descritivos dos clusters. A próxima etapa consiste em verificar se os fatores considerados podem classificar as empresas como pertencente a um cluster específico. Para atingir esse objetivo, foi realizado o procedimento de análise discriminante que, a partir de uma função discriminante, classifica os casos em grupos a partir dos fatores especificados. Foi aplicado o teste de igualdade das médias Wilks'Lambda e, novamente, constatado que "Estratégia inovadora", seguido por "Conhecimento primário e identidade compartilhados" e "Capacidade de absorção de conhecimento", são os fatores mais relevantes no processo de diferenciação, com significância inferior a 0,01. O fator "Sistema de informação" não apresenta diferença estatística significante entre os grupos $(p=0,075)$.

Em relação à capacidade de classificação correta dos casos em função dos clusters formados, a matriz de classificação aponta que a taxa de precisão (hit ratio) é de 93,60\%, uma taxa muito boa, tendo em vista que o mínimo aceitável é $25,00 \%$ a mais do que o acaso - nesse caso, 41,00\% das empresas poderiam ser classificadas corretamente por obra do acaso, e, dessa forma, o mínimo recomendado é de 50,25\% (Hair Jr. et al., 2005). As empresas dos clusters 2 e 4, respectivamente, Inovadoras e Retardatárias, foram classificadas 100,00\% corretamente, ao passo que as empresas do cluster 1 (Explotatoras) e do cluster 3 (Inovadoras) foram classificadas corretamente em 92,00\% e 90,60\% dos casos, respectivamente, pela análise discriminante. 
Tabela 4. Características descritivas dos clusters.

\begin{tabular}{|c|c|c|c|c|}
\hline & Cluster 1 & Cluster 2 & Cluster 3 & Cluster 4 \\
\hline \multicolumn{5}{|l|}{ Tipo de produção } \\
\hline Montadora & 1 & 4 & 2 & 0 \\
\hline Fornecedora autopeça & 31 & 8 & 23 & 9 \\
\hline \multicolumn{5}{|l|}{ Localização da matriz } \\
\hline Ásia & 1 & 0 & 0 & 1 \\
\hline Brasil & 10 & 3 & 8 & 4 \\
\hline EUA & 8 & 3 & 3 & 2 \\
\hline Europa & 13 & 6 & 14 & 2 \\
\hline \multicolumn{5}{|c|}{ Faturamento anual da empresa (em milhões de $R \$$ ) } \\
\hline Acima de 300 & 5 & 6 & 6 & 1 \\
\hline Entre 90 e 300 & 10 & 3 & 9 & 1 \\
\hline Entre 16 e 90 & 17 & 3 & 10 & 7 \\
\hline \multicolumn{5}{|c|}{ Número de funcionários empregados } \\
\hline Acima 4.999 & 3 & 4 & 3 & 0 \\
\hline Entre 1.500 e 4.999 & 6 & 3 & 2 & 1 \\
\hline Entre 500 e 1.499 & 13 & 2 & 9 & 0 \\
\hline Entre 100 e 499 & 10 & 3 & 11 & 9 \\
\hline \multicolumn{5}{|c|}{ Tempo no mercado (anos) } \\
\hline Acima de 40 & 8 & 6 & 10 & 1 \\
\hline Entre 20 e 40 & 10 & 3 & 6 & 2 \\
\hline Entre 10 e 20 & 13 & 1 & 8 & 4 \\
\hline Entre 5 e 10 & 1 & 2 & 1 & 2 \\
\hline
\end{tabular}

Fonte: Elaborada pelos autores (2017).

\section{Discussão}

O desenvolvimento da pesquisa, realizada em empresas do setor automobilístico, revelou a existência de oito fatores contextuais relevantes à GC. Esses fatores constituem o conjunto de requisitos, desenvolvidos no âmbito organizacional, que se relacionam com o ciclo do conhecimento na organização.

Partindo da premissa de que a GC depende de fatores desenvolvidos no âmbito interno das organizações, esta pesquisa estabelece uma tipologia em relação à prática da GC a partir desses fatores contextuais, em empresas do setor automobilístico. Além disso, avalia as implicações das características dos grupos formados nessa tipologia quanto à utilização do conhecimento em atividades que gerem vantagem competitiva.

Os resultados da pesquisa mostram a formação de quatro agrupamentos significativamente diferentes entre si.

O primeiro agrupamento é o que possui o maior número de integrantes (41\%), sendo formado por empresas denominadas de "Explotatoras". Esta denominação deve-se ao fato de que essas organizações desenvolvem mais fortemente os fatores relacionados à resolução de problemas, melhoria incremental e postura proativa, bem como estrutura organizacional enxuta. Em contrapartida, mostra-se reduzido o desenvolvimento de fatores relacionados à capacidade de absorção de conhecimento, estratégia inovadora e até mesmo o desenvolvimento de competências. 
Dessa forma, as organizações do primeiro agrupamento são marcadas por estabilidade do ponto de vista inovativo, e o conhecimento é voltado para a melhoria e aprendizagem incremental. Pode-se constatar também que a maioria dessas empresas (aproximadamente 97\%) é fornecedora de autopeças, grande parte delas de porte intermediário ou pequeno, quando comparadas às demais empresas pesquisadas. Tal constatação sustenta a visão de que esse grupo atua de forma mais "passiva" no mercado, sendo conduzido por montadoras e empresas de autopeças de maior porte no que se refere à inovação e ao avanço tecnológico.

O segundo agrupamento é formado por empresas denominadas de 'Inovadoras', sendo composto por aproximadamente 15\% das organizações pesquisadas. Esse grupo é aquele que contém o maior número de empresas montadoras de veículos automotores - das sete empresas pesquisadas, quatro foram classificadas nesse agrupamento. Também a grande maioria delas possui maior faturamento e maior número de funcionários quando comparadas às demais.

Analisando o desenvolvimento dos fatores, constata-se que essas empresas apresentam os melhores índices, especialmente em relação ao fator "estratégia inovadora" - e também capacidade de absorção, desenvolvimento de competências, conhecimento primário, aprendizagem em grupo e utilização de sistema de informação. Aprofundando a análise sobre o comportamento dos fatores desse grupo, contata-se que as empresas possuem um nível elevado de conhecimento primário, o que corrobora a maior capacidade de absorção e aprendizagem.

Além de maior base de conhecimento primário e de capacidade de absorção e aprendizagem, essas empresas possuem uma estratégia voltada à inovação, acarretando uma maior capacidade de antecipar mudanças, diferenciar e ditar o rumo do mercado e obter maior vantagem competitiva. Portanto, o nível mais elevado dos fatores contextuais leva as empresas desse grupo a desenvolverem de forma mais consistente as fases de aquisição, retenção, distribuição e utilização do conhecimento, referentes ao processo de GC.

O terceiro agrupamento, composto por 32\% das empresas pesquisadas, é formado por empresas denominadas de "Exploradoras". Esse grupo é o segundo dentre aqueles que detêm empresas montadoras (com duas das sete montadoras), ficando atrás apenas do agrupamento "Empresas Inovadoras". A distribuição das empresas em relação ao porte não mostra uma tendência clara de concentração em uma faixa específica.

Em relação aos fatores contextuais, a principal característica é o forte desenvolvimento da capacidade de absorção de conhecimento (o segundo maior índice), bem como o melhor índice em relação ao fator postura proativa e estrutura organizacional enxuta, e o destaque em relação à utilização de sistema de informação - embora associado a um nível modesto quanto ao fator estratégia inovadora.

Tal postura propõe que as organizações desse agrupamento atuam no desenvolvimento de novos conhecimentos e valorizam a atividade explorativa, oferecendo aos funcionários mecanismos para o desenvolvimento de competências e autonomia para a tomada de decisão, evidenciada pelo fator postura proativa e estrutura organizacional enxuta. Os resultados sugerem que tais empresas oferecem aos funcionários uma infraestrutura para o armazenamento do conhecimento e a intensificação da comunicação, como é o caso dos sistemas de informação, porém deixando a cabo das empresas inovadoras, especialmente montadoras e fornecedores estratégicos, o papel de diferenciar o produto no mercado. Portanto, esse grupo de empresas explora o conhecimento a partir de um projeto estabelecido por outras organizações, tornando-as parceiras essenciais.

O quarto agrupamento, constituído por 12\% das empresas pesquisadas, é formado por aquelas denominadas de "Retardatárias". Tal denominação deve-se ao pior desempenho dos fatores contextuais, quando comparados aos demais grupos.

As empresas desse agrupamento apresentam um nível intermediário quanto aos fatores relativos à resolução de problemas e melhoria incremental e desenvolvimento de recursos humanos. Dessa forma, o conhecimento possibilita a essas organizações solucionarem problemas e "apagarem incêndios" inerentes aos processos e, para tanto, apresentam níveis modestos de treinamento e qualificação da mão de obra. 
Em contrapartida, os níveis de aprendizagem, conhecimento primário, capacidade de absorção e, consequentemente, a capacidade inovativa, são bastante reduzidos. Todas as empresas desse agrupamento são fornecedoras de autopeças, sendo a maioria de porte comparativamente inferior às empresas dos demais agrupamentos, seja por faturamento, seja por número de funcionários. Tais características propõem que essas organizações trabalham para outras empresas fornecedoras, sem grande envolvimento em atividade de desenvolvimento, restringindo sua atuação ao âmbito operacional - isto é, executam, por meio de seus processos produtivos, projetos estabelecidos pelos clientes (montadoras ou fabricantes de autopeças). Dessa forma, o papel dessas empresas no setor é acompanhar e apoiar operacionalmente as empresas parceiras (clientes).

A Tabela 5 sintetiza as principais características dos quatro agrupamentos observados nesta pesquisa. A análise discriminante identificou que os quatro agrupamentos são significativamente diferentes entre si. Outra consideração importante resultante desta pesquisa é a análise dos fatores que melhor diferenciam os quatro agrupamentos. "Estratégia inovadora", "Conhecimento primário e identidade compartilhados,, "Postura proativa e estrutura organizacional enxuta" e "Capacidade de absorção de conhecimento" são, em ordem, os quatro fatores com maior capacidade de diferenciar os agrupamentos, isto é, são os fatores que apresentam as maiores diferenças entre os quatro grupos.

Em contrapartida, "Sistema de informação" é o fator com pior capacidade discriminante. O baixo poder discriminatório desse fator evidencia que as tecnologias voltadas aos SI, que apoiam a retenção e distribuição do conhecimento, ou ainda não atingiram a grande adesão por parte das empresas do setor, ou a implantação desses sistemas encontra-se em um quadro de evolução em que as organizações ainda estão se adequando à utilização dessas tecnologias, mesmo nas empresas que constituem os agrupamentos mais evoluídos quanto aos fatores que sustentam a GC.

Tabela 5. Características dos agrupamentos.

\begin{tabular}{|c|c|c|}
\hline Agrupamento & Papel exercido & Principais características \\
\hline Empresas Inovadoras & $\begin{array}{l}\text { Diferenciação; Ditar o rumo } \\
\text { do mercado }\end{array}$ & $\begin{array}{l}\text { Ampla base de conhecimento primário acumulada, sustentada por atividades } \\
\text { de pesquisa; desenvolvimento dos funcionários. A capacidade absortiva e a } \\
\text { aprendizagem apoiam uma estratégia inovativa, o que leva essas empresas a } \\
\text { diferenciarem seus produtos e processos, definindo a dinâmica do mercado. } \\
\text { Utilização de sistemas de informação como suporte à retenção e distribuição } \\
\text { do conhecimento. }\end{array}$ \\
\hline Empresas Exploradoras & $\begin{array}{l}\text { Apoio ao desenvolvimento } \\
\text { das empresas inovadoras }\end{array}$ & $\begin{array}{l}\text { Relevante grau de cumulatividade, originado da capacidade de absorção e } \\
\text { da aprendizagem em grupo associada a uma estratégia inovativa modesta. } \\
\text { Desenvolvimento consistente da mão de obra. Estratégia de desenvolvimento } \\
\text { e pesquisa voltada ao apoio aos projetos de empresas inovadoras. Utilização } \\
\text { de sistemas de informação como suporte à retenção e distribuição do } \\
\text { conhecimento. }\end{array}$ \\
\hline Empresas Retardatárias & Resolução de problemas & $\begin{array}{l}\text { Modesto desenvolvimento em relação à resolução de problemas e melhoria } \\
\text { incremental e desenvolvimento da mão de obra. Os índices dos demais } \\
\text { fatores foram os piores dentre os quatro clusters. Portanto, as empresas desse } \\
\text { agrupamento estão voltadas à solução de problemas ocorridos em âmbito } \\
\text { operacional, sem grandes ambições de explorar novas oportunidades ou apoiar } \\
\text { a estratégia inovativa de empresas parceiras. }\end{array}$ \\
\hline
\end{tabular}




\section{Conclusão}

Considerando o objetivo da pesquisa de estabelecer uma tipologia que caracterize grupos distintos em relação à prática da GC e determine as implicações dessas características quanto à utilização do conhecimento, inicialmente foram levantados os fatores contextuais organizacionais que sustentam o processo de GC.

Os fatores identificados mostram que o processo de GC possui dois elementos centrais. O primeiro é o ser humano, agente responsável por reter e transformar o conhecimento primário por meio de iniciativas de melhoria e inovação. O segundo diz respeito ao contexto organizacional, que deve ser regido por uma cultura que estimule o processo de aprendizagem, e por uma estrutura organizacional que viabilize não só a interação entre as pessoas, mas também o fluxo de informação e conhecimento entre os departamentos.

A partir dos oito fatores identificados na fase inicial, a pesquisa mapeou quatro clusters, compostos por empresas do setor automobilístico (montadoras e fornecedores), com padrões distintos quanto ao desenvolvimento daqueles fatores contextuais que sustentam a GC. Os clusters foram denominados, a partir de seus fatores preponderantes, como "Empresas Inovadoras", "Empresas Exploradoras", "Empresas Explotatoras" e "Empresas Retardatárias".

Pode-se concluir que apenas uma parte dessas empresas, agrupadas como "Inovadoras" e "Exploradoras", em especial as primeiras, utilizam o conhecimento com o objetivo de mudar o padrão tecnológico do mercado. Os outros dois agrupamentos atuam de forma mais passiva e utilizam o conhecimento visando à melhoria da eficiência produtiva ou mesmo à simples resolução de problemas, como fica evidente no agrupamento Retardatário, com baixo desenvolvimento na maioria dos fatores, exceto em 'Resolução de problemas e melhoria incremental'.

As montadoras podem ser consideradas o grupo de empresas "líderes" do setor, pois agem como coordenadoras do processo de desenvolvimento, com o apoio de fornecedores-chave. Esse restrito grupo de empresas, denominados neste trabalho de "Empresas Inovadoras" e "Empresas Exploradoras", tem o papel de inovar, explorar novos conhecimentos e, portanto, de ditar o rumo do desenvolvimento tecnológico do setor. Esses grupos de empresas possuem índices superiores aos demais em relação aos fatores 'conhecimento primário' e 'capacidade de absorção', especialmente o grupo composto por empresas inovadoras, o que implica uma maior capacidade de cumulatividade, isto é, de acumular conhecimento e, consequentemente, desenvolver inovações.

As demais empresas, que constituem os grupos "Empresas Explotatoras" e "Empresas Retardatárias", têm participação mais restrita no setor, quando se avalia a capacidade de adquirir conhecimento e inovar. Essas empresas exercem o papel de apoiar a produção de componentes desenvolvidos pelo primeiro grupo e, portanto, explotam o conhecimento a fim de aperfeiçoar o processo produtivo.

Em relação aos trabalhos futuros, partindo-se dos resultados dos clusters identificados nesta pesquisa, a primeira sugestão é a realização de uma pesquisa qualitativa em empresas pré-selecionadas de cada agrupamento a fim de detalhar os fatores contextuais das empresas. Tomando como referência os fatores e clusters obtidos nesta pesquisa em relação ao segmento automobilístico, sugere-se nova pesquisa em outros setores, a fim de comparar os agrupamentos obtidos, bem como identificar os fatores contextuais que melhor diferenciam os setores pesquisados. Outra possibilidade de trabalho futuro consiste em identificar graus de maturidade em relação à prática da GC. A conclusão deste trabalho expõe que cada cluster identificado apresenta um comportamento distinto frente à estratégia inovativa. Dessa forma, recomenda-se um trabalho que aprofunde o estudo da estratégia inovativa e de sua relação com a GC.

Agradecimentos

À Fundação de Amparo à Pesquisa do Estado de São Paulo (Processos no 2016/24401-2 e 2016/18414-4) e Conselho Nacional de Desenvolvimento Científico e Tecnológico (Processo no 445205/2014-8) pelo apoio financeiro à pesquisa. 
Todos os autores contribuíram na concepção e desenho do estudo, análise de dados e redação final.

\section{Referências}

Associação Nacional dos Fabricantes de Veículos Automotores. Anuário estatístico da indústria automotiva brasileira. São Paulo: ANFAVEA, 2016. Disponível em: <http://www.anfavea. com.br>. Acesso em: 1 ago. 2016.

Chen, C. J.; Huang, J. W. How organizational climate and structure affect knowledge management: The social interaction perspective. International Journal of Information Management, v. 27, n. 2, p. 104-18, 2007.

Chen, C. J; Huang, J. W.; Siao, Y. C. Knowledge management and innovativeness: The role of organizational climate and structure. International Journal of Manpower, v. 31, n. 8, p. 48-70, 2010.

Cohen, W. M.; Levinthal, D. A. Absorptive Capacity: A new perspective on learning and innovation. Administrative Science Quarterly, v. 35, p. 128-152, 1990.

Corfield, A.; Paton, R. Investigating knowledge management: can KM really change organisational culture? Journal of Knowledge Management, v. 20, n. 1, p. 88-103, 2016.

Costello, A. B.; Osborne, J. W. Best practices in exploratory factor analysis: Four recommendations for getting the most from your analysis. Practical Assessment, Research and Evaluation, v. 10, n. 7, p. 1-9, 2005.

Cross, R.; Sproull, L. More than an answer: Information relationships for actionable knowledge. Organization Science, v, 15, n. 4, 446-462, 2004.

Forza C. et al. research in operations management: a processbased perspective. International Journal of Operations and Production Management, v. 22, n. 2, p. 152-194, 2002.

Ganzaroli, A. et al. The combined effect of technological relatedness and knowledge utilization on explorative and exploitative invention performance post-M \& A. European Journal of Innovation Management, v. 19, n. 2, p. 167-188, 2016.

Garicano, L.; Wu, Y. Knowledge, communication, and organizational capabilities. Organization Science, v. 23, n. 5, p. 1382-1397, 2012.

Gonzalez, R. V. D.; Martins, M. F. Mapping the organizational factors that support knowledge management in the Brazilian automotive industry. Journal of Knowledge Management, v. 18, n. 1, p. 152-176, 2014.

Gonzalez, R. V. D.; Martins, M. F.; Toledo, J. C. Managing knowledge in a service provider: A network structure-based model. Journal of Knowledge Management, v. 18, n. 3, p. 611-630, 2014

Grant, R. M. Toward a knowledge-based theory of the firm. Strategic Management Journal, v. 17, p. 109-122, 1996.

Hair Jr. et al. Multivariate data analysis. New Jersey: Prentice Hall, 2005.
Hwang, E. H.; Sinh, P.V.; Argote, L. Knowledge sharing in online communities: Learning to cross geographic and hierarchical boundaries. Organization Science, v. 26, n. 6, p. 1593-1611, 2015.

Irani, Z.; Sharif, A. M.; Love, P. E. D. Mapping knowledge management and organizational learning in support of organizational memory. International Journal of Production Economics, v. 122, p. 200-215, 2009.

Kane, G. C.; Alavi, M. Information technology and organizational learning: An investigation of exploration and explotation process. Organization Science, v. 18, n. 5, p. 796-812, 2007.

Lee, P. K. C.; To, W. M.; Ty, B. T. W. Team attributes and performance of operational service teams: An empirical taxonomy development. International Journal of Production Economics, v. 142, p. 51-60, 2013.

Lefebvre, V. M. et al. Social capital and knowledge sharing performance of learning networks. International Journal of Information Management, v. 36, p. 570-579, 2016.

Liao, C.; Chuang, S. H.; To, P. L. How knowledge management mediates relationship between environment and organizational structure. Journal of Business Research, v. 64, p. 728-736, 2011.

Lin, H. F. A stage model of knowledge management: An empirical investigation of process and effectiveness. Journal of Information Science, v. 33 n. 6, p. 643-659, 2007.

Lin, T. C.; Chang, C. L. H.; Tsai, W. C. The influences of knowledge loss and knowledge retention mechanisms on the absorptive capacity and performance of a MIS department. Management Decision, v. 54, n. 7, p. 1757-1787, 2016.

López-Sáez, P. et al. External knowledge acquision processes in knowledege-intensive clusters. Journal of Knowledge Management, v. 14, n. 5, p. 690-707, 2010.

Magnier-Watanable, R.; Senoo, D. Organizational characteristics as prescriptive factors of knowledge iniciatives. Journal of Knowledge Management, v. 12, n. 1, p. 21-36, 2008.

March, J. G. Exploration and exploitation in organizational learning. Organization Science, v. 20, n.1, p. 71-87, 1991.

Marouf, L. The role of knowledge sharing culture in business performance. VINE Journal of Information and Knowledge Management Systems, v. 46, n. 2, p. 154-174, 2016.

Martins, E. C.; Meyer, H. W. J. Organizational and behavioral factors that influence knowledge retention. Journal of Knowledge Management, v. 16. n. 1, p. 77-96, 2012.

Massaro, M. et al. Knowledge management in small and medium enterprises: A structured literature review. Journal of Knowledge Management, v. 20, n. 2, p. 258-291, 2016. 
Mingoti, S. A. Análise de dados através de métodos de estatística multivariada: uma abordagem aplicada. Belo Horizonte: Editora UFMG, 2005.

Patterson, W.; Ambrosini, V. Configuring absorptive capacity as a key process for research intensive firms. Technovation, v. 36-37, p. 77-89, 2015.

Preacher, K. J.; MacCallum, R. C. Exploratory factor analysis in behavior genetics research: Factor recovery with small sample sizes. Behavior Genetics, v. 32, n. 2, p. 153-161, 2002.

Quigley, N. R. et al. A multilevel investigation of the motivational mechanisms underlying knowledge sharing and performance. Organization Science, v. 18, n. 1, p. 71-88, 2007.

Teece, D. J. Explicating dynamic capabilities: The nature and microfondations of (sustainable) enterprise performance. Strategic Management Journal, v. 28, p. 1319-1350, 2007.

Torugsa, N. A.; O'Donohue, W. Progress in innovation and knowledge management research: From incremental to transformative innovation. Journal of Business Research, v. 69, p. $1610-1614,2016$.
United Nations Conference on Trade and Development. Word Investment Report 2005: Transnational corporations and the internationalization of R\&D. New York: United Nations, 2005.

Van Dijk, A.; Hendriks, P.; Romo-Leroux, I. Knowledge sharing and social capital in globally distributed execution. Journal of Knowledge Management, v. 20, n. 2, p. 327-343, 2016.

White, G. R. T.; Cicmil, S. Knowledge acquisition through process mapping: Factors affecting the performance of work-based activity. International Journal of Productivity and Performance Management, v. 65, n. 3, p. 302-323, 2016.

Yang, J. The knowledge management strategy and its effect on firm performance: A contingency analysis. International Journal of Production Economics, v. 125, n. 2, p. 215-223, 2010.

Zangiski, M. A. S. G.; Lima, E. P.; Costa, S. E. G. Organizational competence building and development: Contributions to operations management. International Journal of Production Economics, v. 144, n. 1, p. 76-89, 2013. 